\title{
Transfer-matrix renormalization group study of the spin ladders with cyclic four-spin interactions
}

\author{
H. T. Lu, ${ }^{1,2}$ L. Q. Sun, ${ }^{2}$ Shaojin Qin, ${ }^{2}$ Y. J. Wang ${ }^{3}$ \\ ${ }^{1}$ School of Physics, Peking University, Beijing 100871, China \\ ${ }^{2}$ Institute of Theoretical Physics and Interdisciplinary Center of Theoretical Studies, \\ Chinese Academy of Sciences, Beijing 100080, China \\ ${ }^{3}$ Department of Physics, Beijing Normal University, Beijing 100875, China
}

\begin{abstract}
The temperature dependence of the specific heat and spin susceptibility of the spin ladders with cyclic four-spin interactions in the rung-singlet phase is explored by making use of the transfermatrix renormalization group method. The values of spin gap are extracted from the specific heat and susceptibility, respectively. It is found that for different relative strength between interchain and intrachain interactions, the spin gap is approximately linear with the cyclic four-spin interaction in the region far away from the critical point. Furthermore, we show that the dispersion for the one-triplet magnon branch can be obtained by numerically fitting on the partition function.
\end{abstract}

PACS numbers: 75.10.Jm, 75.40.Cx, 75.40.Mg

\section{INTRODUCTION}

In the past few years, cyclic spin exchanges in quantum antiferromagnetic magnets have attracted considerable attention. From various experiments, it has been realized that four-spin cyclic exchanges play an important role in understanding the spin dynamics of insulating cuprate materials, such as the two-leg spin ladders $\mathrm{Cu}_{2} \mathrm{O}_{3}$ in the compounds $\mathrm{SrCu}_{2} \mathrm{O}_{3}, \frac{1}{,}$ ( $\left.\mathrm{Sr}, \mathrm{Ca}, \mathrm{La}\right)_{14} \mathrm{Cu}_{24} \mathrm{O}_{41}, \frac{2,3,4}{2}$ and the $\mathrm{CuO}_{2}$ plane in $\mathrm{La}_{2} \mathrm{CuO}_{4} \stackrel{5.6}{\underline{6}}$ Similar mechanism of multiple spin exchanges in ${ }^{3} \mathrm{He}$ (Ref:7) and Wigner crystal,,$\frac{8}{}$ which was found to be large, has also been proposed.

Spin ladders can be viewed as intermediates between one-dimensional spin chains and two-dimensional spin lattices. They are particular of interest in the understanding of the high-temperature superconductivity in two dimensions and the Haldane's conjecture $\frac{9,10}{}$ in spin chains. The two-leg spin ladder is the simplest system on which cyclic exchanges can be materialized. In the absence of cyclic exchanges, with nonzero interchain couplings, the $S=1 / 2$ spin ladder lies in a Haldane phase, which has a short-range resonating-valence-bond (RVB) ground state with a spin gap, and superconductivity emerges upon doping $\underline{11,12}$ Contrary to the case in a $S=1 / 2$ chain, the spinons in the ladder are confined back to the well-defined quasiparticles (massive magnons) in the neighborhood of $q=\pi$, where a spectral gap exists $\stackrel{13}{\underline{13}}$ It has been shown that four-spin cyclic exchanges can make the spin gap decreases rapidly $\underline{\underline{2}}$ Furthermore, tuning of the four-spin interactions can drive the system into different phases $\stackrel{14,15}{=}$ At the nearest critical point, the system becomes gapless to cross from the RVB rung-singlet phase to a staggered dimer phase. By a field theory using the Majorana Fermion representation, Nersesyan and Tsvelik ${ }^{16}$ referred to the critical phenomenon as the level $k=2 \mathrm{SU}(2)$ Wess-Zumino-NovikovWitten model or $\mathrm{SU}(2) c=\frac{3}{2}$ conformal field theory. In other words, the phase transition is of the secondorder type and belongs to the universality class of the

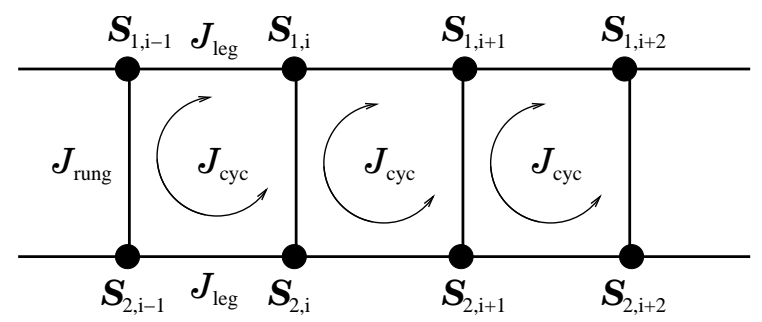

FIG. 1: Schematic structure of a two-leg ladder with cyclic (four-spin) interaction. The round arrows stand for the action of the four-spin terms as given in Eq. (1), which is not completely identical to the usual cyclic permutations.

Takhtajan-Babujian critical point $\underline{17}$ This statement has been confirmed by numerical simulations $\frac{18,19}{19}$

The Hamiltonian describing an isotropic $S=1 / 2$ spin ladder with additional cyclic (four-spin) interaction is represented by

$$
\begin{aligned}
H & =\sum_{i}\left\{J_{\text {rung }} \mathbf{S}_{1, i} \cdot \mathbf{S}_{2, i}+J_{\text {leg }}\left[\mathbf{S}_{1, i} \cdot \mathbf{S}_{1, i+1}+\mathbf{S}_{2, i} \cdot \mathbf{S}_{2, i+1}\right]\right. \\
& +2 J_{\text {cyc }}\left[\left(\mathbf{S}_{1, i} \cdot \mathbf{S}_{1, i+1}\right)\left(\mathbf{S}_{2, i} \cdot \mathbf{S}_{2, i+1}\right)\right. \\
& +\left(\mathbf{S}_{1, i} \cdot \mathbf{S}_{2, i}\right)\left(\mathbf{S}_{1, i+1} \cdot \mathbf{S}_{2, i+1}\right) \\
& \left.\left.-\left(\mathbf{S}_{1, i} \cdot \mathbf{S}_{2, i+1}\right)\left(\mathbf{S}_{2, i} \cdot \mathbf{S}_{1, i+1}\right)\right]\right\}
\end{aligned}
$$

where the indices 1 and 2 distinguish upper and lower legs, and $i$ labels rungs; $J_{\text {rung }}$ and $J_{\text {leg }}$ are the interchain and intrachain couplings, respectively; $J_{\text {cyc }}$ denotes the strength of the cyclic (four-spin) exchange. The model is schematically shown in Fig. 1. In the rest of the paper, for convenience, we call Eq. (1) as type-I Hamiltonian.

The above Hamiltonian distinguishes from another one where the ring exchange term is directly represented by a cyclic permutation operator $P_{i j k l}$ in the plaquette:

$$
K\left(P_{i j k l}+P_{i j k l}^{-1}-\frac{1}{4}\right) .
$$


We call this form as type-II Hamiltonian. The cyclic permutation operator can be expressed by spin- $\frac{1}{2}$ operators including bilinear frustrating and biquadratic terms $\stackrel{2,20}{=}$ So type-II Hamiltonian can be re-written as

$$
\begin{aligned}
H & =\sum_{i}\left\{\left(J_{\text {rung }}+2 K\right) \mathbf{S}_{1, i} \cdot \mathbf{S}_{2, i}\right. \\
& +\left(J_{\text {leg }}+K\right)\left(\mathbf{S}_{1, i} \cdot \mathbf{S}_{1, i+1}+\mathbf{S}_{2, i} \cdot \mathbf{S}_{2, i+1}\right) \\
& +K\left(\mathbf{S}_{1, i} \cdot \mathbf{S}_{2, i+1}+\mathbf{S}_{2, i} \cdot \mathbf{S}_{1, i+1}\right) \\
& +4 K\left[\left(\mathbf{S}_{1, i} \cdot \mathbf{S}_{1, i+1}\right)\left(\mathbf{S}_{2, i} \cdot \mathbf{S}_{2, i+1}\right)\right. \\
& +\left(\mathbf{S}_{1, i} \cdot \mathbf{S}_{2, i}\right)\left(\mathbf{S}_{1, i+1} \cdot \mathbf{S}_{2, i+1}\right) \\
& \left.\left.-\left(\mathbf{S}_{1, i} \cdot \mathbf{S}_{2, i+1}\right)\left(\mathbf{S}_{2, i} \cdot \mathbf{S}_{1, i+1}\right)\right]\right\}
\end{aligned}
$$

There have been extensive theoretical studies on the type-II Hamiltonian. By the density matrix renormalization group (DMRG) $\underline{11}$ and exact diagonalization method, the energy spectra about one-magnon branch and twomagnon continuum in the rung-singlet phase have been obtained $\stackrel{4.10}{=}$ The phase diagram for $J_{\text {rung }}=J_{\text {leg }}$ was studied in detailed 14 and a global picture for general cases has been proposed. $\stackrel{15}{\underline{n}}$ The phase transitions, mainly concerning the case from the rung-singlet to the dimerized phase, together with the critical exponent were studied by field theory, perturbative and series expansion method $\frac{18,19,20,22}{2}$

On the other hand, the type-I Hamiltonian catches less attention. And it is widely believed that the physics should be the same for the type-I and type-II Hamiltonians, although there is a lack of detailed comparisions. Theoretically, the ring exchange interaction naturally arises as an effective low-energy model for the multiband Hubbard model near the insulating filling and in the strong coupling limit. It has been shown that the four-spin terms are most significant ones $\frac{23}{2}$ In order to highlight the effect of this four-spin term, in this paper, we employ the type-I Hamiltonian for our numerical simulations.

As mentioned above, the zero-temperature behaviors of the spin ladders with cyclic exchange interactions have been analyzed in detail, especially for the type-II Hamiltonian. A study on the thermodynamic properties of the systems has not been carried out extensively, except for the study by a high-temperature series expansion method $\stackrel{24}{=}$ These quantities can be directly compared with the experimental observations and therefore are of particular use. Detailed and reliable numerical results on these quantities may offer an expedient way to determine the model parameters for the compounds we are interested in. In this paper, we show that by using the transfer-matrix renormalization group (TMRG) method, we can not only obtain the temperature dependence of the specific heat and susceptibility of the spin ladders down to the low-temperature regime and with high accuracy, but also extract extra information from the data for the magnitude of the gap and the one-triplet magnon excitations, which can be compared with the experiments directly.
As proposed by experiments, for the insulator cuprate ladder materials, $\underline{1,2.3 .4 .25}$ both $J_{\text {rung }}$ and $J_{\text {leg }}$ are positive and close in magnitude. The values of $K / J_{\text {rung }}$ range in a region $0.025 \leq K / J_{\text {rung }} \leq 0.075$, which roughly corresponds to the parameter region $0.05 \leq J_{\text {cyc }} / J_{\text {rung }} \leq 0.15$ for the type-I Hamiltonian and the system is still in the rung-singlet phase. For this reason, in our numerical simulations, we confine the parameter $x_{\mathrm{cyc}} \in[0,0.1]$, and $x$ is taken three different values: $0.5,1.0$ and 1.2 , where

$$
\begin{aligned}
x & :=J_{\text {leg }} / J_{\text {rung }}, \\
x_{\text {cyc }} & :=J_{\text {cyc }} / J_{\text {rung }} .
\end{aligned}
$$

We take $x=0.5$ in order to make a comparison with the typical rung-singlet phase.

The numerical algorithm we employ to study the thermodynamical properties of the present model is the TMRG method, 26,27,28 which is a powerful numerical tool for studying the thermodynamic properties of onedimensional quantum systems. One is able to evaluate nearly all thermodynamic quantities by the maximum eigenvalue and the corresponding left and right eigenvectors of the transfer matrix. We will show later that the magnitude of the gap and the low-lying excitations can also be extracted from the obtained data. In our numerical calculations, $\tau=0.05$, the error caused by the Trotter-Suzuki decomposition is less than $10^{-3}$. During the TMRG iterations, $80-100$ states are retained and the truncation error is less than $10^{-4}$ down to $k_{B} T \sim 0.01 J_{\text {rung }}$.

\section{SPECIFIC HEAT AND SPIN SUSCEPTIBILITY}

Figure 2 shows the TMRG results on the temperature dependence of the specific heat coefficient $C / T$ for $x=$ $0.5,1.0,1.2$ with $x_{\mathrm{cyc}}=0,0.02,0.04,0.06,0.08$ and 0.1 . Besides the exponential decrease as $T \rightarrow 0$, which indicates the existence of a gap, the general behavior of the curves for a given $x$ is as follows: As $x_{\text {cyc }}$ increased, the slope of $(C / T)_{\max }$ shifts to lower temperatures. The reason is obvious: that with the increase of the four-spin cyclic exchange, the spin gap and the whole dispersion decrease $\stackrel{2.22}{=}$ Another interesting feature is that at $x=$ 1.0 and 1.2 , a shoulder gets more and more distinct at low temperatures with the increase of $x_{\mathrm{cyc}}$. We attribute this shoulder to the tendency of deconfinement of bound spinons from the magnons, as the crossover occurs from the strong coupling regime $\left(J_{\text {leg }} / J_{\text {rung }}=x \ll 1\right)$ to the weak coupling one $\left(J_{\operatorname{leg}} / J_{\text {rung }}=x \gg 1\right)$ with the extreme limiting case of a finite $C / T$ value at $T=0$.

The corresponding results for spin susceptibility $\chi(T)$ are presented in Fig. 3. The exponential decrease of $\chi(T)$ as $T \rightarrow 0$, and the shift of the peak to lower temperatures with the increase of $x_{\text {cyc }}$ are consistent with the above behaviors of $C / T$. Our numerical result on the susceptibility well agrees with that of Ref. 24 obtained 


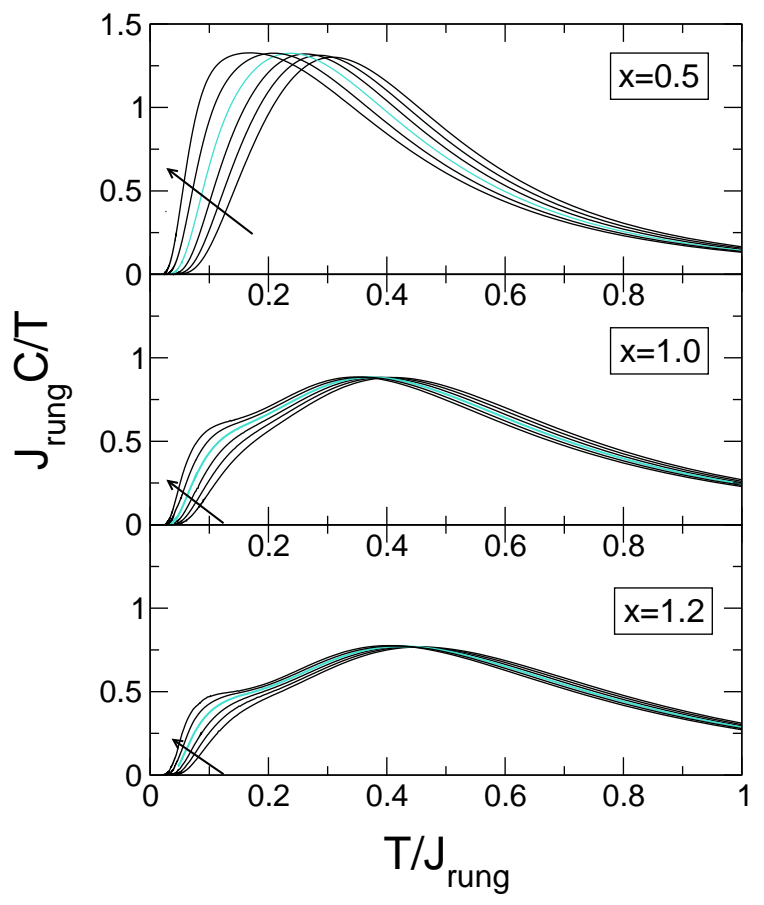

FIG. 2: (Color online) The specific heat coefficient $C / T$ for type-I Hamiltonian for three values of $x$ with $x_{\text {cyc }}=0,0.02$, $0.04,0.06,0.08$, and 0.1 , in ascending order along the direction of arrows.

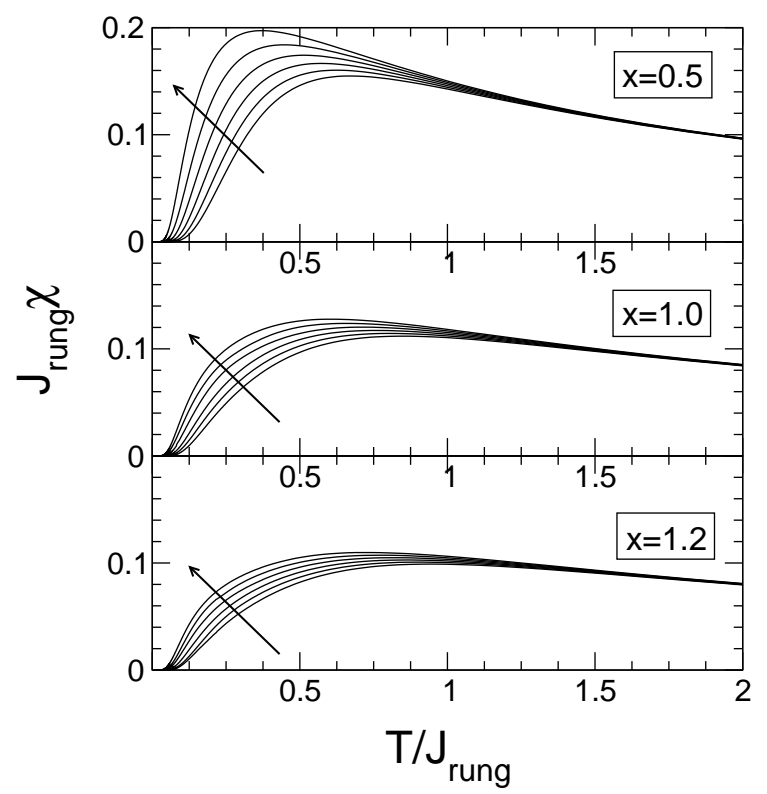

FIG. 3: Temperature dependence of the susceptibility $\chi$ for type-I Hamiltonian for three values of $x$ with $x_{\text {cyc }}=0,0.02$, $0.04,0.06,0.08$, and 0.1 in ascending order along the direction of arrows.

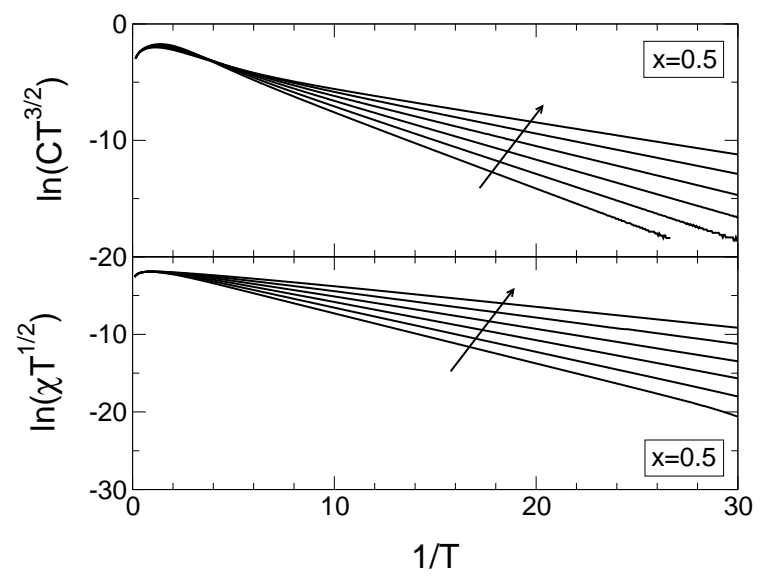

FIG. 4: $1 / T$ dependence of $\ln \left(C T^{3 / 2}\right)$ and $\ln \left(\chi T^{1 / 2}\right)$ (in the unite of $\left.J_{\text {rung }}\right)$ for $x=0.5$ with $x_{\text {cyc }}=0,0.02,0.04,0.06$, 0.08 , and 0.1 in ascending order along the direction of arrows.

by high-temperature series expansion and exact diagonalization.

The $x_{\text {cyc }}$ dependence of the gap is a central quantity, since basically it determines the relevancy of the cyclic exchange term. In the vicinity of the critical point $x_{\mathrm{cyc}}^{c}$, generally,

$$
\Delta \sim\left|x_{\mathrm{cyc}}-x_{\mathrm{cyc}}^{c}\right|^{\eta}
$$

For the type-II Hamiltonian in both strong and weak coupling limits, by the perturbative cluster expansion and Padé approximation in the strong coupling regime, together with the bosonization technique in the weak coupling limit, Müller et al $\stackrel{20}{\underline{2}}$ showed that the critical exponent $\eta$ for the gap is approximately equal to 1 ; i.e., the gap is linearly dependent on $x_{\text {cyc }}$ near the critical point. For the $J_{\text {leg }}=J_{\text {rung }}$ case, this result was also obtained by Hijii et al $\underline{\underline{19}}$ Here, we will see that for type-I Hamiltonian, in the region considerably far away from the critical point, $x_{\text {cyc }} \in(0,0.1)$, the linear behavior of the gap vs. $x_{\text {cyc }}$ still holds in almost all the parameter range.

For the spin- $\frac{1}{2}$ ladder in the rung-singlet phase, similar to the case in a $S=1$ chain, we can assume that the low-lying excitation spectrum $\epsilon(k)$ for $k$ near $\pi$ is approximately given by

$$
\begin{aligned}
\epsilon(k) & =\sqrt{v^{2}(k-\pi)^{2}+\Delta^{2}} \\
& \approx \Delta+\frac{v^{2}}{2 \Delta}(k-\pi)^{2}+\mathcal{O}\left(|k-\pi|^{3}\right),
\end{aligned}
$$

where $v$ is the spin wave velocity and $\Delta$ is the spectrum gap. The low temperature behaviors of the specific heat and susceptibility per spin can be expressed, respectively, $\operatorname{as}^{29}$ 


$$
\begin{aligned}
& C(T) \approx \frac{3 \Delta}{v \sqrt{8 \pi}}\left(\frac{\Delta}{T}\right)^{3 / 2} e^{-\Delta / T}, \\
& \chi(T) \approx \frac{1}{v} \sqrt{\frac{\Delta}{2 \pi T}} e^{-\Delta / T},
\end{aligned}
$$

for $T \ll \Delta$. In this situation, $\ln \left(C T^{3 / 2}\right)$ and $\ln \left(\chi T^{1 / 2}\right)$ should be linear to $1 / T$ with a slope $-\Delta$. Figure 4 shows the $1 / T$ dependence of $\ln \left(C T^{3 / 2}\right)$ and $\ln \left(\chi T^{1 / 2}\right)$ (in units of $\left.J_{\text {rung }}\right)$ for $x=0.5$ with $x_{\text {cyc }}=0,0.02,0.04$, $0.06,0.08$, and 0.1 . We observe a well-behaved linearity at $1 / T>10$. For $x=1.0$ and 1.2 , we have the similar results. The values of the gap at various $x$ and $x_{\text {cyc }}$ can thus be extracted by fitting the data in the interval $\beta=1 / T \in(10,20)$. The results for $x=0.5$ and $x=1.0$ are presented in Fig. 5. The gaps obtained from the specific heat and susceptibility are well coincident with each other.

To both verify the low-temperature scaling behavior of the system and check the validity of Eq. (7), in Fig. 6, the rescaled susceptibility $\chi / \chi_{\max }$ versus $T / \Delta$ for $x=0.5$, 1.0 at various $x_{\text {cyc }}$ is presented. For a fixed value of $x$, the curves converge to a single one at low temperatures, demonstrating the dominance of $\Delta$ in determining the susceptibility at low temperatures. Furthermore, it also suggests that the values of the gap we obtained by this approach are quantitatively reliable. This situation is quite similar to the case of the $S=1$ antiferromagnetic Heisenberg chain with single-ion anisotropy $\stackrel{30}{=}$ Here $\chi_{\max }$ serves as a common factor which incorporates the unknown effect on the prefactor in Eq. (7).

From Fig. 5, we observe that the gap is approximately linearly dependent on $x_{\text {cyc }}$ for fixed $x$ in the parameter space we studied. We also notice the different descending rates of $\Delta$ with $x_{\text {cyc }}$ for various $x$. At $x_{\text {cyc }}=0, \Delta(x=0.5)>\Delta(x=1.0)$ with the difference $\sim 0.15$. This indicates that the gap decreases as the intrachain coupling $J_{\text {leg }}$ increases, which is consistent with the perturbative picture from the dimer limit of uncoupled rungs (strong coupling regime). On the other hand, at $x_{\text {cyc }}=0.1$, the difference of the gaps becomes very small. We expect for higher value of $x_{\text {cyc }}$ a reverse of the gaps with $\Delta(x=0.5)<\Delta(x=1.0)$ should occur.

For $x=1.0$ and 1.2 (not shown in Fig. 5), the values of the gap obtained by the present method well coincide with the numerical results from the density-matrix renormalization group (DMRG) study for the type-II Hamiltonian for the corresponding values of the cyclic exchange coupling, $\stackrel{41}{, 11}$ For instance, when $x=1.0$ and $x_{\text {cyc }}=0$, the gap obtained here by TMRG is $\sim 0.51$, as compared with the DMRG result of 0.504 .11

To come to a close, we estimates the errors in the process of gap fitting. On the one hand, as mentioned in the previous section, combining the errors from the TrotterSuzuki decomposition with the TMRG truncation, we estimate that the error caused by the TMRG algorithm in the fitting interval (in our work $T \in(0.05,0.1)$ ) is less

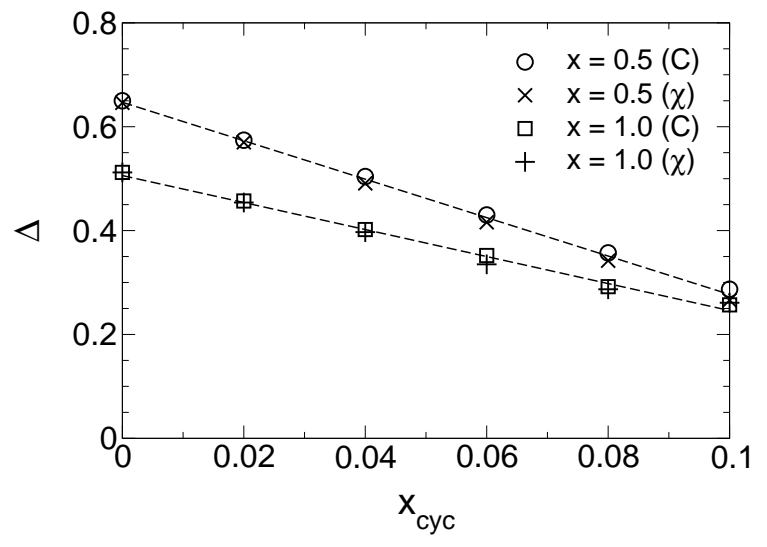

FIG. 5: The $x_{\text {cyc }}$ dependence of the spin gap for $x=0.5$ and 1.0 obtained by fitting the Eqs. (6) (signed as $C$ ) and (7) (given as $\chi$ ), respectively.

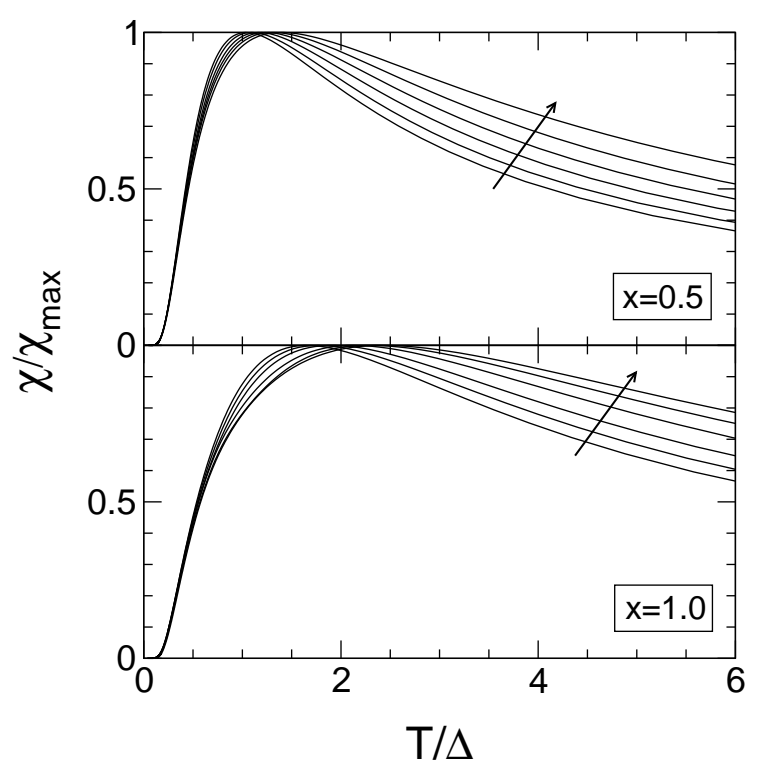

FIG. 6: $\chi / \chi_{\max }$ versus $T / \Delta$ for $x=0.5,1.0$ with $x_{\text {cyc }}=0$, $0.02,0.04,0.06,0.08$, and 0.1 in ascending order along the direction of arrows.

than $10^{-2}$ at most. On the other hand, in obtaining the fitting formulas (6) and (7), the low-temperature limit and quadratic approximation (See Eq. (5)) have been adopted. For the specific heat, the expression including the higher order contributions of $T / \Delta$ is given explicitly as 31

$$
\begin{aligned}
C(T)= & \frac{3 \Delta}{v \sqrt{8 \pi}}\left(\frac{\Delta}{T}\right)^{3 / 2} \\
& \times\left[1+\frac{T}{\Delta}+\frac{3}{4}\left(\frac{T}{\Delta}\right)^{2}\right] e^{-\Delta / T} .
\end{aligned}
$$


The uncertainty in Eq. (6) is roughly estimated as

$$
\delta \Delta \sim \bar{T} \ln \left[1+\frac{\bar{T}}{\Delta}+\frac{3}{4}\left(\frac{\bar{T}}{\Delta}\right)^{2}\right],
$$

where $\bar{T}$ represents some value of temperature $T$ lying in the fitting interval. From this, in our case the uncertainty of gap due to the fitting formulas can be estimated approximately as $\delta \Delta \approx 0.01-0.02$. Based on these considerations, we conclude that the error in the gap fitting is about $10^{-2}$, which is not exceeding the symbol size in Fig. 5 .

\section{PARTITION FUNCTION AND THE DISPERSION RELATIONS}

In TMRG calculations, the partition function $Z_{\mathrm{TMRG}}(\beta)$ is the original and the most accurate numerical quantity. In previous section we obtained the specific heat and spin susceptibility, which can also be got from the derivatives of the partition function directly. In the following we will discuss the single-particle state in this system and show that its dispersion, as well as the ground-state energy and the magnitude of the gap, can be obtained from the analyses of the partition function.

In the rung singlet phase, the elementary excitations are gapped. The energy gap and dispersion for the single particle state can be analyzed perturbatively ${ }^{20}$ When $J_{\text {leg }}=J_{\text {cyc }}=0$, the system is consisted of an array of rungs with a vacuum state being the product of spin singlet state on each rung. Above this vacuum state, a hard core boson of spin 1 is the only possible excited state on each rung. The system has a flatband dispersion $\epsilon(k)=J_{\text {rung }}$ for single particle excitations. Due to the hard core scattering between the bosons, the statistics of the system is essentially Fermi-Dirac type,,$\underline{32}$ which allows us to write down the partition function for the system:

$$
\ln Z_{\mathrm{eff}}(\beta)=\frac{1}{2 \pi} \int_{-\pi}^{\pi} \ln \left(1+3 e^{-\beta \epsilon(k)}\right) d k-\beta e_{0},
$$

where $e_{0}$ is the ground-state energy per rung in the thermodynamic limit and the prefactor 3 before $e^{-\beta \epsilon(k)}$ comes from the three internal states of the single particle excitations.

In the rung singlet phase, switching upon the couplings $J_{\text {leg }}$ and $J_{\text {cyc }}$ will induce a dispersion ${ }^{4,10,20}$ for the single particle state, and the interaction between the particles. Classified by the internal symmetries, the two-particle interaction is either spin-channel independent or spinchannel dependent. A bound state may also appear in certain spin-channels. This has been observed in a previous study,,$\underline{4}$ in which it is shown that the bound state is also momentum dependent. Therefore, the interaction is very complicated and depends on the total momentum of the scattering particles.

Since we are dealing with a system with massive particles at zero chemical potential, the density of the particles

\begin{tabular}{cccccccc}
\hline \hline$x_{\text {cyc }}$ & $\mu_{0}$ & $\mu_{1}$ & $\mu_{2}$ & $\mu_{3}$ & $e_{0}$ & $\Delta$ & $\Delta_{\mathrm{C}}$ \\
\hline 0 & 1.58 & 0.59 & -0.38 & 0.09 & -1.156 & 0.53 & 0.51 \\
\hline 0.02 & 1.57 & 0.60 & -0.39 & 0.10 & -1.137 & 0.47 & 0.46 \\
\hline 0.04 & 1.55 & 0.62 & -0.41 & 0.12 & -1.119 & 0.42 & 0.40 \\
\hline 0.06 & 1.54 & 0.63 & -0.42 & 0.13 & -1.101 & 0.37 & 0.35 \\
\hline 0.08 & 1.53 & 0.64 & -0.43 & 0.15 & -1.084 & 0.31 & 0.30 \\
\hline 0.1 & 1.52 & 0.65 & -0.44 & 0.15 & -1.068 & 0.27 & 0.26 \\
\hline \hline
\end{tabular}

TABLE I: The partition function fitting results by Eq. (10) for the $x=1.0$ case. $\Delta_{\mathrm{C}}$ in the last column is the energy gap obtained by Eq. (6), as plotted in Fig. 5 .

would be dilute at low temperatures. Taking into account of the form of the effective partition function for single particle dispersion $Z_{\text {eff }}$, we expect it would match the accurate TMRG results at both high and low temperatures by including higher order harmonics in the dispersion: ${ }^{31}$

$$
\epsilon(k)=\mu_{0}+\mu_{1} \cos (k)+\mu_{2} \cos (2 k)+\mu_{3} \cos (3 k) .
$$

The energy gap is given by $\Delta=\mu_{0}-\mu_{1}+\mu_{2}-\mu_{3}$ at $k=\pi$. Starting with a set of trial values for $\mu$ 's given by perturbative calculation,,$\underline{\underline{ }}$ we minimize the squared 2 -norm of the residual in free energy calculated from the trial dispersion:

$$
\begin{aligned}
& W\left(\mu_{0}, \mu_{1}, \mu_{2}, \mu_{3}, e_{0}\right)= \\
& \sum_{i=1}^{N}\left[\ln Z_{\text {eff }}\left(\beta_{i}\right)-\ln Z_{\mathrm{TMRG}}\left(\beta_{i}\right)\right]^{2},
\end{aligned}
$$

where $\beta_{i}$ are smoothly distributed in the interval $(5,15)$ with a spacing $\Delta \beta=0.05$. Typically, we show the fitting results for the $x=1.0$ case in Table $\square$ The corresponding dispersions are plotted in Fig. 7 In our final numerical results, the values of $W$ in Eq. (12) are of order $10^{-6}$, and the mean deviation at each data point is less than $10^{-4}$.

From Table \ we find that $\mu_{0}$ and $\mu_{1}$ monotonically decreases and increases with $x_{\mathrm{cyc}}$, respectively. This is consistent with the perturbative results $\underline{20}$ On the other hand, the third order coefficient $\mu_{3}$ increases with $x_{\text {cyc }}$. At $x_{\text {cyc }}=0.1$, it reaches 0.15 , about one-third of $\mu_{2}$. This indicates that the approximation up to the third order in the dispersion is necessary for $x=1.0$ case. On the contrary, for $x=0.5, \mu_{0} \sim 1, \mu_{1} \sim 0.5-0.6$, the absolute value of both $\mu_{2}$ and $\mu_{3}$ are less than 0.1 , and the dispersion can be satisfactorily approximated by singlecomponent harmonics with only $\mu_{1}$. This fact reflects that with the increase of $J_{\text {leg }}$ and $J_{\text {cyc }}$, higher orders in the harmonic expansion are needed to describe the oneparticle excitation in the spectrum.

The dispersion relations shown in Fig. 7 should correspond to the one-triplet magnon branch in the spectrum of the ladder. We compare the results with the previous studies by the DMRG $\stackrel{4,10}{=}$ We find they have quite similar 


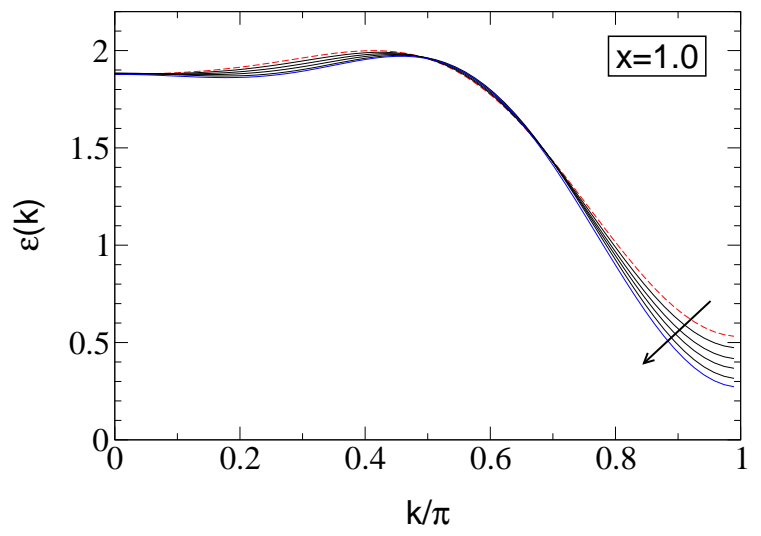

FIG. 7: (Color online) Dispersion (in units of $J_{\text {rung }}$ ) for $x=$ 1.0 with $x_{\mathrm{cyc}}=0,0.02,0.04,0.06,0.08$, and 0.1 in descending order along the direction of arrows.

shapes and are quantitatively agreement in the vicinity of $k=\pi$. The deviation when approaching $k=0$ can be attributed partially to the two-triplet bound state arising from the magnon-magnon interaction that we have ignored $\underline{\underline{4}}$

The energy gaps obtained in this way are presented in Table I. and are compared with their counterparts from the specific heat data. We see that they are rather coincident. In addition, we have obtained the ground state energy ( $e_{0}$ in Eq. (10) $)$, which is compatible with the results from the zero-temperature DMRG scheme.

\section{SUMMARY}

In this paper, we explored the thermodynamic properties of the spin ladders with cyclic four-spin interactions by the TMRG method. The temperature dependences of the specific heat and the susceptibility of the ladder in the rung-singlet phase are analyzed numerically. Based on the above results, we extracted the values of the spin gap and found that it manifests approximately linear behavior with the cyclic interaction in the parameter space we considered. We also showed that the dispersion for low-lying excitations can be obtained from the partition function very effectively by combining with the meanfield or perturbative studies. Comparing with the high temperature series expansion and the exact diagonaliza-

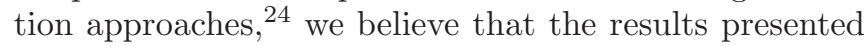
in this study give more direct and accurate physical quantities in the thermodynamic limit. Further experimental results on specific heat and susceptibility may allow one to determine the various parameters for the two-leg spin ladder materials via this approach. In addition, the dispersion relations extracted from the free energy may be used to be compared with the experimental data of the inelastic neutron scattering spectra.

\section{Acknowledgments}

1 A. Gößling, U. Kuhlmann, C. Thomsen, A. Löffert, C. Gross, nd W. Assmus, Phys. Rev. B 67, 052403 (2003).

2 S. Brehmer, H.-J. Mikeska, M. Müller, N. Nagaosa, and S. Uchida, Phys. Rev. B 60, 329 (1999).

${ }^{3}$ M. Matsuda, K. Katsumata, R. S. Eccleston, S. Brehmer, and H-J. Mikeska, Phys. Rev. B 62, 8903 (2000); M. Matsuda, K. Katsumata, R. S. Eccleston, S. Brehmer, and H-J. Mikeska, J. Appl. Phys. 87, 6271 (2000).

4 T. S. Nunner, P. Brune, T. Kopp, M. Windt, and M. Grüninger, Phys. Rev. B 66, R180404 (2002).

5 Y. Honda, Y. Kuramoto, and T. Watanabe, Phys. Rev. B 47, 11329 (1993).

${ }^{6}$ R. Coldea, S. M. Hayden, G. Aeppli, T. G. Perring, C. D. Frost, T. E. Mason, S.-W. Cheong, and Z. Fisk, Phys. Rev. Lett. 86, 5377 (2001).

7 G. Misguich, B. Bernu, C. Lhuillier, and C. Waldtmann, Phys. Rev. Lett. 81, 1098 (1998).

8 S. Chakravarty et al. Philos. Mag. B 79, 859 (1999); M. Katano and D. S. Hirashima, Phys. Rev. B 62, 2573 (2000); B. Bernu, Ladir. Cândido, and D. M. Ceperley,
Phys. Rev. Lett. 86, 870 (2001).

9 F. D. M. Haldane, Phys. Lett. 93A, 464 (1983); Phys. Rev. Lett. 50, 1153 (1983); J. Appl. Phys. 57, 3359 (1985).

10 G. Roux, S. R. White, S. Capponi, A. Läuchli, and D. Poilblanc, Phys. Rev. B 72, 014523 (2005).

11 S. R. White, R. M. Noack, and D. J. Scalapino, Phys. Rev. Lett. 73, 886 (1994).

12 E. Dagotto and T. M. Rice, Science 271,618 (1996), and references therein.

13 D. G. Shelton, A. A. Nersesyan, and A. M. Tsvelik, Phys. Rev. B 53, 8521 (1996).

14 A. Läuchli, G. Schmid, and M. Troyer, Phys. Rev. B 67, R100409 (2003).

15 V. Gritsev, B. Normand, and D. Baeriswyl, Phys. Rev. B 69, 094431 (2004).

16 A. A. Nersesyan and A. M. Tsvelik, Phys. Rev. Lett. 78, 3939 (1997).

17 P. P. Kulish, N. Y. Reshetikhin, and E. K. Sklyanin, Lett. Math. Phys. 5, 393 (1981); L. A. Takhtajan, Phys. Lett. 87A, 479 (1982); H. M. Babujian, ibid. 90A, 479 (1982). 
18 K. Hijii and K. Nomura, Phys. Rev. B 65, 104413 (2002).

19 K. Hijii, Shaojin Qin, and K. Nomura, Phys. Rev. B 68, 134403 (2003).

20 M. Müller, T. Vekua, and H.-J. Mikeska, Phys. Rev. B 66, 134423 (2002).

${ }^{21}$ S. R. White, Phys. Rev. Lett. 69, 2863 (1992); Phys. Rev. B 48, 10345 (1993).

${ }^{22}$ K. R. Schmidt, H. Monien, and G. S. Uhrig, Phys. Rev. B 67, 184413 (2003).

${ }^{23}$ E. Müller-Hartmann and A. A. Reischl, Eur. Phys. J. B 28, 173 (2002).

24 A. Bühler, U. Löw, K. P. Schmidt, and G. S. Uhrig, Phys. Rev. B 67, 134428 (2003).

25 M. Windt, M. Grüninger, T. Nunner, C. Knetter, K. P. Schmidt, G. S. Uhrig, T. Kopp, A. Freimuth, U. Ammerahl, B. Büchne, and A. Revcolevschi, Phys. Rev. Lett. 87, 127002 (2001).
26 R. J. Bursill, T. Xiang, and G. A. Gehring, J. Phys.: Condens. Matter 8, L583 (1996).

27 X. Wang and T. Xiang, Phys. Rev. B 56, 5061 (1997).

28 T. Xiang and X. Wang, in Density-Matrix Renormalization: A New Numerical Method in Physics, edited by I. Peschel, X. Wang, M. Kaulke, and K. Hallberg (Springer, New York, 1999), pp. 149-172.

29 T. Xiang, Phys. Rev. B 58, 9142 (1998).

30 D. Coombes, T. Xiang, and G. A. Gehring, J. Phys.: Condense. Matter 10, L159 (1998).

31 M. Troyer, H. Tsunetsugu, and D. Würtz, Phys. Rev. B 50, 13515 (1994).

32 Jizhong Lou, Shaojin Qin, Tai-Kai Ng, Zhaobin Su, and Ian Affleck, Phys. Rev. B 62, 3786 (2000); K. Okunishi, ibid. 60, 4043 (1999). 